\title{
Clinical Implications of the Stress Response
}

\author{
George Minowada*‡ and William J. Welch $\$$ s \\ ${ }^{*}$ Division of Pulmonary Medicine and Departments of ${ }^{\ddagger}$ Medicine and ${ }^{\S}$ Physiology, University of California, San Francisco, \\ San Francisco, California 94143-0854
}

The heat shock response, first observed in Drosophila melanogaster over thirty years ago, provided investigators a relatively simple way to study rapid changes in gene expression. Simply raising the temperature of Drosophila above its physiologic norm resulted in the decreased expression of those genes which were active before the temperature shock and the increased expression of genes encoding a group of proteins referred to as the heat shock proteins (hsp's). ${ }^{1}$ Over the last 30 years similar changes in gene expression following relevant temperature shocks have been observed in cells from all organisms, be they derived from bacteria, plants, yeast, or mammals. Moreover, the heat shock proteins from various organisms appear highly conserved with respect to their primary structure, mode of regulation, and biochemical function. In addition to heat shock treatment, many other types of metabolic insults including exposure to heavy metals, amino acid analogs, different metabolic poisons as well as a variety of relevant insults in vivo (e.g., ischemia/reperfusion) also elicit increased expression of the hsp's. Accordingly, many investigators now refer to the response more generally as the stress response, and the proteins whose expression increases, the stress proteins.

As one might predict, the stress response represents a universally conserved cellular defense program. Perhaps the best example of how the stress response provides for increased cellular protection is illustrated by the phenomenon of "acquired thermotolerance." Cells subjected to a sublethal heat shock treatment, if provided a subsequent recovery period at their normal growth temperature, now are able to survive a second and what would otherwise be a lethal heat shock challenge. Acquired thermotolerance is usually transient, lasting about 24 $\mathrm{h}$ in cells grown in culture, and appears dependent upon a number of changes induced by the initial or "priming" heat shock treatment, including the increased expression and accumulation of the stress proteins. Moreover, we now know that any particular agent or treatment which results in an induction of the stress

Address correspondence to William J. Welch, Division of Pulmonary Medicine, Departments of Medicine and Physiology, University of California, San Francisco, San Francisco, CA 94143-0854. Phone: 415-4768546; 415-206-4123. 1994.

Received for publication 18 April 1994 and in revised form 7 June

1. Abbreviations used in this paper: hsp, heat shock protein; HSF, heat shock factor; grp, glucose regulated protein.

J. Clin. Invest.

(c) The American Society for Clinical Investigation, Inc. 0021-9738/95/01/0003/10 \$2.00

Volume 95, January 1995, 3-12 response will confer added protection to the cell upon its exposure to a subsequent and unrelated stressor (e.g., prior heat shock treatment provides for protection to sodium arsenite treatment and vice versa). We also now know that despite their designation as heat shock or stress proteins, most of these proteins are in fact expressed constitutively in normal or "unstressed" cells where they play a fundamental role in a number of important biological processes. For example, many of the constitutively expressed hsp's function as so-called "molecular chaperones," facilitating various aspects of protein maturation throughout the cell. The stress response/proteins also play an important role in a number of clinically relevant phenomenon including tissue and organ trauma, and the immune response. Here we will discuss the clinical implications of the heat shock response and stress proteins beginning with a brief summary of the structure/function of the hsp's and then turn toward more recent developments with potential clinical ramifications. (Because of space limitation, we cannot cite all of the appropriate references, and therefore, refer the reader to other recent reviews whenever possible).

\section{Induction of the heat shock response}

As mentioned above, a diverse array of metabolic insults including the exposure of cells to elevated temperatures, heavy metals, various ionophores, amino acid analogs, and metabolic poisons which target ATP production, all result in similar changes in gene expression leading to the accumulation of the stress proteins. Many of these agents/treatments which induce a stress response share the common property of being "protein chaotropes;" they adversely affect the proper conformation and therefore the function of proteins. Consequently, it was proposed (1) and later demonstrated (2) that under conditions where abnormally folded proteins might begin to accumulate in the cell, a stress response would be initiated. Presumably the resultant increases in the levels of the stress proteins would somehow facilitate the identification, removal, and/or restoration of those proteins adversely affected by the particular stress event, again a prediction which now appears correct (1). The intracellular accumulation of abnormally folded proteins initiates the stress response by somehow activating a specific transcription factor, referred to as the heat shock factor (HSF-1) (for review see reference 3). HSF-1, present in the normal, unstressed cell as an inactive monomer, rapidly trimerizes in response to metabolic stress. Trimerization enables HSF-1 to bind to a consensus nucleotide sequence, referred to as the heat shock element (HSE), located within the promoter element of those genes encoding the stress proteins thereby resulting in the high level transcription of the heat shock genes. Recently, another related transcription factor, HSF-2, has been identified. Shown to be important in regulating the expression of hsp tran- 
Table I.

\begin{tabular}{|c|c|c|c|c|}
\hline Name & Size* & Bacterial homologue & Locale & Remarks \\
\hline & $k D$ & & & \\
\hline Ubiquitin & 8 & - & Cytosol/nucleus & Involved in nonlysosomal protein degradation pathway \\
\hline Hsp 10 & 10 & Gro ES & Mitochondria/chloroplast & Cofactor for Hsp 60 \\
\hline $\begin{array}{l}\text { Low molecular } \\
\text { weight hsp's }\end{array}$ & $20-30$ & $\begin{array}{l}\text { Possible homologues } \\
\text { recently identified }\end{array}$ & Cytosol/nucleus & $\begin{array}{l}\text { Proposed regulator of actin cytoskeleton; proposed } \\
\text { molecular chaperone }\end{array}$ \\
\hline Hsp 47 & 47 & - & Endoplasmic reticulum & Collagen chaperone \\
\hline Hsp 56 & 56 & - & Cytosol & Part of steroid hormone receptor complex; binds FK506 \\
\hline Hsp 60 & 60 & Gro EL & Mitochondria/chloroplast & Molecular chaperone ("chaperonin") \\
\hline TCP-1 & 60 & Gro EL & Cytosol/nucleus & Molecular chaperone related to Hsp 60 \\
\hline Hsp 72 & 70 & Dna K & Cytosol/nucleus & Highly stress inducible \\
\hline Hsp 73 & 70 & Dna K & Cytosol/nucleus & Constitutively expressed molecular chaperone \\
\hline Grp 75 & 70 & Dna $\mathrm{K}$ & Mitochondria/chloroplast & Constitutively expressed molecular chaperone \\
\hline Grp 78 (BiP) & 70 & Dna $\mathbf{K}$ & Endoplasmic reticulum & Constitutively expressed molecular chaperone \\
\hline Hsp 90 & 90 & htpG & Cytosol/nucleus & $\begin{array}{l}\text { Part of steroid hormone receptor complex; chaperone (?) for } \\
\text { retrovirous-encoded tyrosine protein kinases }\end{array}$ \\
\hline Hsp 104/110 & $104 / 110$ & Clp family & Cytosol/nucleus & Required to survive severe stress; molecular chaperone (?) \\
\hline
\end{tabular}

* Approximate size by SDS-page, native molecular weight often is very different.

scription during hemin-induced differentiation of K562 cells (4), HSF 2 also may turn out to be important for controlling the activities of hsp gene expression in the normal or unstressed cell.

\section{Overview of the structure and function of the stress proteins}

The mammalian stress proteins often are divided into two groups based on their classical mode of induction: the heat shock proteins (hsp's) and the glucose regulated proteins (grp's) (for review see reference 5 and 6). In general, both the hsp's and grp's are identified and referred to on the basis of their apparent molecular mass as determined by SDS-PAGE (Table I). Members of the grp family were first observed to exhibit increased expression in cells starved of glucose. Subsequently, the grp's were shown to also undergo high level expression in cells treated with calcium ionophores, when subjected to anoxic-like conditions, or in response to added reducing agents such as B-mercaptoethanol. Interestingly, many of the agents/treatments which induce the grp's adversely affect protein secretion. Perhaps not too surprising, the major grp's reside within the endoplasmic reticulum and participate in various aspects of protein secretion. We now know that the grp's are in fact related to members of the hsp family, and that the two families of stress proteins exhibit considerable similarities with respect to both their structure and function.

Our understanding of the structure and function of the stress proteins has advanced rapidly in recent years, sometimes from unexpected sources. For example, several proteins whose function had already been defined, subsequently were shown to be synthesized at higher levels in the cell experiencing stress (for review see reference 6 ). Ubiquitin, a small polypeptide of $\sim 8$ $\mathrm{kD}$ and known to be involved in targeting proteins for degradation, is synthesized at relatively high rates after heat shock. Hsp56, characterized initially as part of a larger protein complex that binds steroid hormone receptors (for review see reference
7), and now known to bind to the immunosuppressants FK506 (8) and rapamycin, shows increased expression after stress (9). Like other identified FK506 binding proteins, hsp56 appears to function as a peptidylprolyl cis-trans isomerase (rotamase). Other examples of proteins whose function was already known and subsequently shown to represent members of the stress protein family include: heme oxygenase, involved in catalyzing the breakdown of heme into biliverdin; the multiple drug resistance gene product or $P$-glycoprotein, a plasma membrane transporter involved in multidrug resistance to many cancer chemotherapeutic agents; alpha B-crystallins, integral structural components of the lens and now known to be present in other cell types; and at least two glycolytic enzymes enolase and glyceraldehyde 3-phosphate dehydrogenase. For at least some of these proteins we have some insights as to why they are upregulated after stress. For example, the glycolytic pathway provides an essential energy source in the event that aerobic respiration becomes uncoupled, often a result of metabolic stress. Consequently, increases in the synthesis of the aforementioned glycolytic enzymes may facilitate the increased demand on the glycolytic pathway in the cell under stress. Similarly, higher levels of ubiquitin may provide for the cell under stress an increased capacity to recognize and degrade irreparably damaged proteins. We suspect that other proteins whose function have already been established, also will be found to be stress inducible, perhaps in a cell type specific manner.

As is presented in Table $\mathrm{I}$, the number of proteins whose expression increases in eukaryotic cells after metabolic stress is rather extensive and continues to grow. Instead of providing an overview of all of these proteins, we will focus our discussion on those stress proteins which have been best characterized and/ or which have generated the most surprises as it relates to their structure/function. Perhaps the most exciting development involves the role of some of the stress proteins in facilitating protein biogenesis (for reviews see references 10 and 11). Here two families of stress proteins, the hsp60 and hsp70 families, 
each consisting of multiple and related members which are expressed constitutively in all cells, have been shown to participate directly in various aspects of protein maturation. Members of the hsp70 family, distributed throughout various subcellular compartments and expressed in cells grown under normal conditions include: hsp73 (a k a constitutive hsp70, hsc70, or hsp70 cognate), present within the cytoplasm and nucleus; grp75, a component of the mitochondria; and grp78 (a k a BiP) a resident of the endoplasmic reticulum (ER). In addition, under conditions of metabolic stress another form of hsp70, referred to as the highly stress-inducible hsp70 (or hsp72), is synthesized at very high levels, exhibits considerable homology to hsp73, and like hsp73 resides within the nucleus and cytoplasm. Although too extensive to discuss here, other proteins related to the hsp70 family also have been identified, but their function remains somewhat obscure. All members of the hsp70 family have been shown to bind ATP through a highly conserved amino-terminal nucleotide binding domain, whose overall structure appears very similar to that of two other ATP binding proteins, actin and hexokinase. In addition, presumably via their carboxy-terminal domain, hsp70 family members appear to bind to both unfolded proteins and short polypeptides in vitro. Results from many laboratories have shown that members of the hsp70 family, within their own distinct subcellular compartment, interact with other cellular proteins undergoing synthesis on the ribosome or translocation into organelles. These observations have led to the suggestion that the hsp70 family members function in the early stages of protein maturation by binding to and stabilizing the unfolded state of a newly synthesized protein. Once synthesis or organellar translocation of the target protein has been completed, the particular hsp70 family member is released, in a process requiring ATP and likely other proteinaceous cofactors, thereby allowing the target protein to commence folding and/or assembly.

In a similar manner, members of the hsp60 family also bind ATP, and interact transiently with unfolded polypeptides (for review see references 10 and 11). In plant and animal cells related forms of hsp60 have been observed in both mitochondria and chloroplasts. Like their bacterial counterpart, the GroEL protein, members of the hsp60 family are characterized by their distinctive seven-membered ring-like structure, often being found in vivo as two rings stacked one on top of the other. In addition to binding to unfolded proteins, members of the hsp60 family (also referred to as chaperonins) appear to "catalyze" protein folding and/or protein assembly. In conjunction with a co-factor of $\sim 10 \mathrm{kD}$ (referred to as GroES in bacteria), which like hsp60 assembles into a seven-membered ring particle, hsp60 binds to an unfolded protein and facilitates the subsequent folding of the target protein. Recently, electron microscopy studies have revealed that the target protein undergoing folding is actually present within the central cavity of the hsp60 "double-donut." It has been suggested that the target protein, via multiple rounds of release and rebinding, likely fueled by ATP hydrolysis, eventually acquires its final folded structure. Once properly folded the protein no longer appears as a target for binding to the particular chaperonin (12). As one might expect, other related members of the hsp60 family are rapidly being identified. For example, recent studies indicate that there may be as many as 8 or more hsp60-like homologs (referred to as the TCP-1 family) within the cytoplasm of yeast and animal cells (13). To date however, no chaperonin equivalent has been identified in the secretory pathway.

These observations regarding the structure and apparent function of both hsp60 and hsp70 have led to new proposals regarding the mechanisms of protein folding/assembly. Earlier work by Anfinsen (14) and others demonstrated the principle of protein "self-assembly," all of the information necessary for the proper folding of a protein is provided by its primary amino acid sequence. This conclusion was based on the simple but elegant observations that a protein, when denatured by a protein chaotrope (e.g., urea), could spontaneously refold upon removal of the chaotrope. Results from studies of the hsp60 and hsp70 families have led to a modification of these earlier concepts. In vivo protein folding and/or assembly may in fact occur by "assisted self-assembly," with the assistance provided by so-called "molecular chaperones" such as members of the hsp60 and 70 families (for reviews see references 15 and 11). Molecular chaperones function in ways that do not contradict the principle of self-assembly. For example, while not conveying any specific information for folding, molecular chaperones participate in the process by preventing improper or nonproductive intra- or inter-molecular interactions that could lead to protein misfolding and/or aggregation. Molecular chaperones do not become a part of the final, properly folded protein. By facilitating productive folding and assembly pathways, molecular chaperones ensure high fidelity and efficiency in the protein folding/assembly process. In fact, many investigators have suggested that molecular chaperones like hsp70 and hsp60 may work in tandem to facilitate the folding process. For example, as a newly synthesized protein emerges from the ribosome the nascent chain is stabilized in an unfolded state via its interaction with cytosolic hsp 70. After completion of its synthesis the polypeptide either commences directly along its folding pathway or alternatively, is transferred over to a member of the chaperonin family where folding and/or higher ordered assembly takes place (Fig. 1). In a similar scenario, as a newly synthesized protein begins its entry from the cytoplasm into an organelle, it does so in a relatively unfolded state, likely mediated via cytosolic hsp 70 . As the protein enters into the organelle it now is recognized by the organellar form of hsp 70 and thereby is maintained in the unfolded conformation. Once translocation has been completed and now with all of the information for folding being present the polypeptide either spontaneously folds or more likely, is transferred over to a member of the chaperonin family where either folding and/or assembly with other polypeptides commences (Fig. 2).

In perhaps a variation of the chaperone concept, another constitutively expressed stress protein, hsp90, also appears to interact transiently with at least certain proteins. For example, during the course of examining the maturation pathway of $\mathrm{pp} 60^{\mathrm{src}}$, the virally encoded tyrosine protein kinase of Rous sarcoma virus, investigators observed that newly synthesized pp60 $0^{\mathrm{src}}$ interacted with two cytosolic proteins, one being hsp90 and the other an unidentified $50-\mathrm{kD}$ component. While present in such a complex in the cytoplasm, pp60 $0^{\mathrm{src}}$ appeared inactive, unable to function as a tyrosine protein kinase. Once the complex reached the inner side of the plasma membrane, $\mathrm{pp} 60^{\mathrm{src}}$ was released and became partially integrated into the plasma membrane where it now appeared biologically active. Although less developed, a similar maturation pathway appears to characterize several other retrovirus-encoded oncogenic tyrosine pro- 

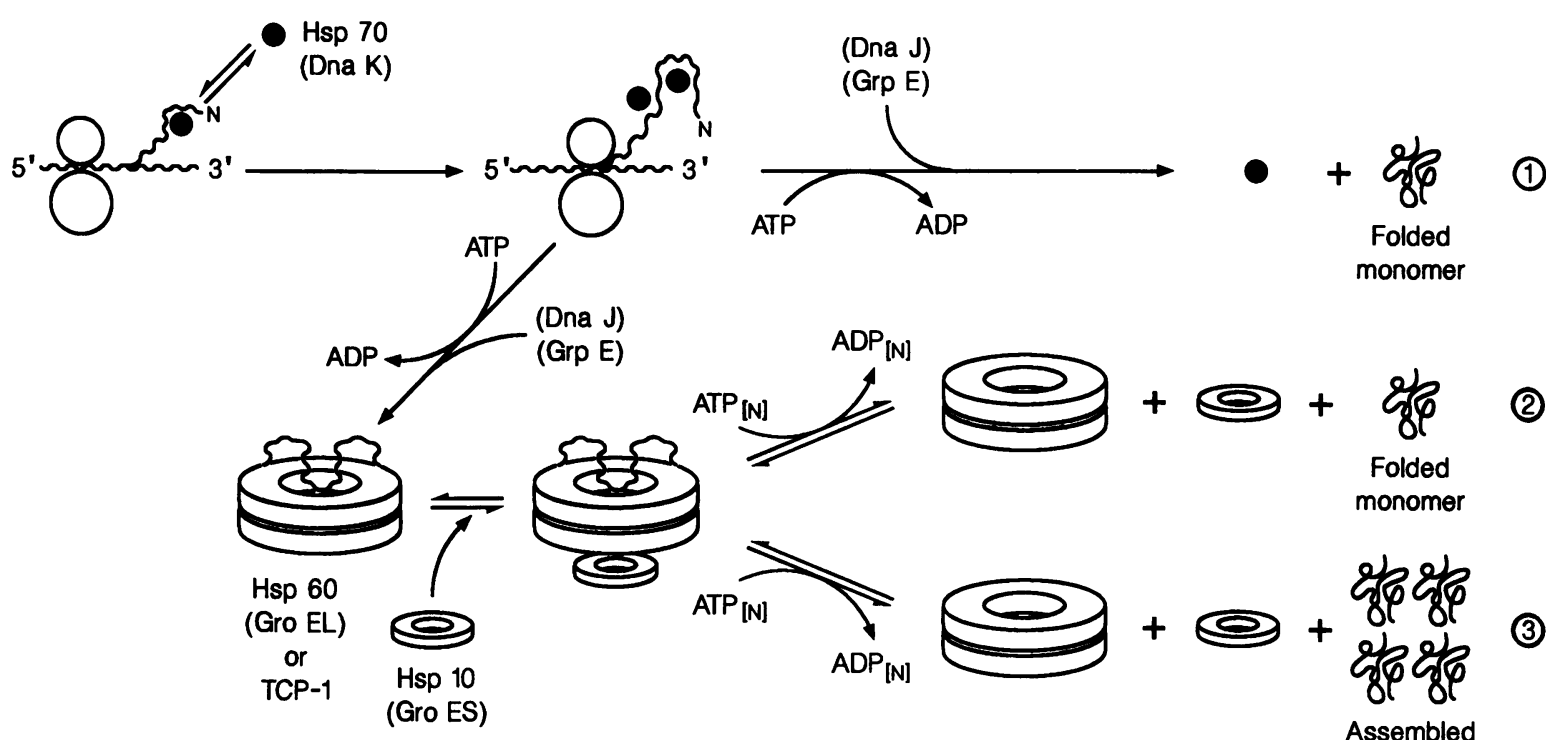

\section{(1)}
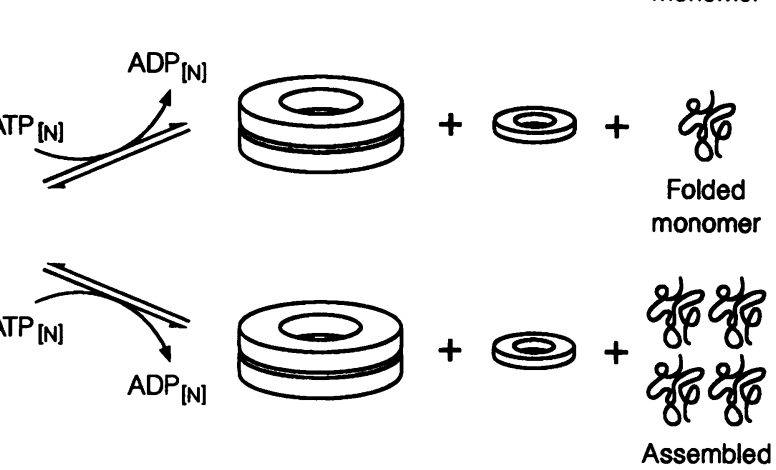

oligomer

Figure 1. Current models by which molecular chaperones facilitate protein folding/assembly. During the course of protein synthesis, Hsp70 (DnaK) interacts with the nascent chain as it emerges from the ribosome. Such an interaction may prevent the premature folding of the nascent chain and/ or prevent its inappropriate interaction with components of the translational machinery. For some proteins, folding of the completed polypeptide may commence upon its release from the Hsp70 chaperone and may not require any additional components. Release of the Hsp70 chaperone likely is mediated by additional factors such as DnaJ and GrpE (pathway 1). Alternatively, following their synthesis, some newly synthesized proteins, perhaps still bound to Hsp70 (DnaK), are transferred to the particular chaperonin (Hsp60, GroEL, TCP-1). Again, release and/or transfer of the nascent polypeptide from its Hsp70 chaperone likely requires the participation of additional components (DnaJ and GrpE). Binding of the unfolded polypeptide to the chaperonin triggers the recruitment of Hsp 10 (GroES) to the opposite side of the chaperonin complex (it is possible that Hsp10/ GroES may already be occupying this end of the chaperonin before substrate binding). Folding of the polypeptide, probably within the cavity of the chaperonin, may involve a series of binding and release events of the substrate, facilitated by many rounds of ATP hydrolysis, until folding is completed (pathway 2). Finally, it remains possible that the Hsp60 (GroEL), or TCP-1 chaperonins, preferentially participate in the folding and/ or assembly of only those proteins that are part of homo- or hetero-oligomeric structures (pathway 3). Reproduced, with permission, from the Annual Review of Cell Biology, Volume 9, (C) 1993, by Annual Reviews Inc.

tein kinases (for review see reference 16). To date, the exact biological role served by hsp90 in the proper maturation and/ or regulation of these various protein kinases remains unclear. However, hsp90, in conjunction with several other members of the stress protein family (e.g., hsp70, hsp56), also has been shown to interact with various steroid hormone receptors, intracellular proteins which when activated by their appropriate steroid ligand now become active as a transcription factor (for review see reference 17 ). Similar to the situation with the virally encoded protein kinases, steroid receptors appear to be maintained in an inactive state when bound to an oligomeric protein complex that includes hsp90. Binding of the steroid hormone appears to initiate a series of events wherein hsp90 is released from the complex, enabling the receptor to properly oligomerize and thereby acquire a DNA binding conformation. These observations in sum, point toward a role for hsp90 in regulating the biological activities of target proteins with whom it transiently interacts. In particular, hsp90 may bind to or mask domains of target proteins which are critical for their biological activation and/or function.

Recently, members of the low molecular weight hsp's (molecular masses of 20-30 kD), were reported to exhibit molecular chaperone-like properties, facilitating at least in vitro, the accelerated folding of a target polypeptide (18). However, unlike the other more well defined chaperones (e.g. hsp 60 and hsp 70), the low-molecular-weight hsp's do not appear to bind nucleotides like ATP. Yet other studies have concluded that the low-molecular-weight hsp's may be important regulatory components of the actin based cytoskeleton. For example, the single low molecular weight hsp in mammalian cells, hsp28, has been reported to act as both an inhibitor of actin polymerization, as well as promote the disassembly of already formed actin filaments in vitro (19). Overexpression of mammalian hsp28 in vivo resulted in an apparent stabilization of and/or increase in the actin containing stress fibers (20). Moreover in the overexpressing cells, a sub population of hsp28 was shown to colocalize with that portion of actin present within the "ruffling" membrane present at the leading edge of the cell (21). Because so many of the agents (including heat shock treatment, a wide variety of cytokines, mitogens, and tumor promoters) that induce the rapid phosphorylation of hsp28 also induce a rapid rearrangement of the actin cytoskeleton, particularly the cortical actin network underlying the plasma membrane, the hypothesis that hsp28 somehow is involved in regulating the actin cytoskeleton remains attractive and is under further study by a number of laboratories.

Our progress in elucidating the functions of the stress proteins in the normal cell also have been enlightening in terms of our understanding why many of these proteins are upregulated in cells undergoing metabolic stress. Specifically, under conditions in which protein folding is perturbed, or pre-existing proteins begin to unfold and denature (e.g., in response to heat 


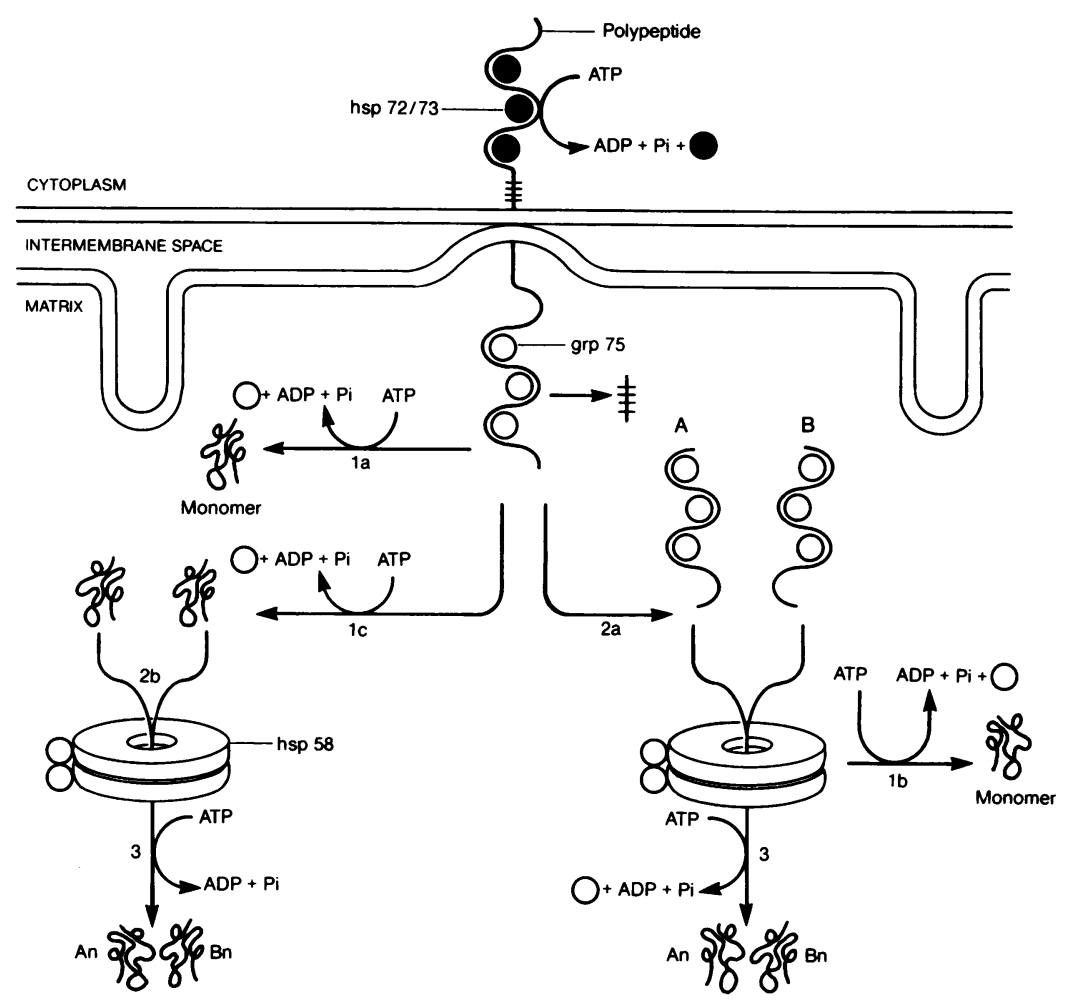

Figure 2. A model describing the possible role of grp 75 and hsp 60 (hsp 58) in mitochondrial protein import, folding, and assembly. Newly synthesized proteins destined for the mitochondria are maintained in an unfolded or translocation competent state within the cytoplasm by virtue of their interaction with the cytosolic hsp 72/ 73. Translocation of the polypeptide into the mitochondria is accompanied by the ATP-dependent release of hsp 72/73. As the translocating and unfolded polypeptide enters into the mitochondria, it becomes complexed with grp 75, and the mitochondrial signal sequence is removed by signal peptidase. Once entirely inside the mitochondria, folding of the polypeptide commences, accompanied by the ATP-dependent release of grp 75 (la). For some monomeric mitochondrial proteins, it remains possible that folding is also dependent on an interaction with hsp $58(1 b)$. For the assembly of oligomeric proteins, grp 75 is released from the monomer (1c); the monomer then moves to hsp $58(2 b)$ and is assembled into its oligomeric form (3). Alternatively, the monomer, still bound to grp 75 , moves to hsp 58 $(2 a)$ and is assembled into its oligomeric form (3). Reproduced from Cell Regulation, 1991, 2:165-179 by copyright permission of the American Society for Cell Biology. shock treatment), the cell responds by increasing the synthesis of stress proteins, many of which function as molecular chaperones. Presumably the resultant increased levels of the stress proteins affords the cell a means by which to $(a)$ identify and perhaps facilitate refolding of those proteins adversely affected by the metabolic insult; $(b)$ identify and bind to abnormally folded proteins for their eventual targeting to an appropriate proteolytic system; $(c)$ facilitate the synthesis and maturation of new proteins needed to replace those which were adversely affected during the particular metabolic insult. We suspect that all three of these possible scenarios are likely correct. At the cellular level however, the exact role of the stress proteins as it pertains to the acquisition of cellular thermotolerance remains less well defined. Controversy exists as to which members of the stress protein family are the most important "contributors" for the overall protection of the cell during the acquisition of thermotolerance. Moreover, whether other physiologic changes which accompany the induction of the stress response are also important for the development of the thermotolerant state continues to be debated. Our own prejudice is that increases in the levels of the stress proteins are in fact important, but by no means the sole basis by which to explain the complicated phenomenon of cellular thermotolerance.

\section{Clinical implications of the stress response/proteins}

Induction of the stress response in vivo. Are the biochemical and functional characteristics of the heat shock response/proteins as defined by cell culture experiments relevant to organs and tissues in the whole organism? Examination of tissues and organs subjected to various metabolic insults such as ischemia or fever revealed that the stress response does occur in different tissues/ organs in vivo (for review see reference 6). In addition, it has been observed that seizures and excitatory amino acids such as glutamate can induce a stress response in the brain $(22,23)$. Increased synthesis of stress proteins was also observed in the rat heart subjected to hemodynamic overload (24). These findings that physiologically relevant insults can induce the stress response in vivo have led to studies examining whether the phenomenon of cellular thermotolerance also is operative in the animal. Preliminary work from a number of labs appears promising in this regard. For example, rodents subjected to whole body hyperthermia (which was demonstrated to result in increased levels of the stress proteins in the heart) suffered less myocardial damage in response to a subsequent ischemia/ reperfusion episode $(25)$. Similar results were found in a rabbit model (26). In contrast, Yellon et al. (27) did not observe such a protective effect, but their study used a longer ischemia/ reperfusion insult. Taken together, these studies indicate that hyperthermic treatment can render the myocardium more resistant to ischemia-reperfusion-induced damage, but only up to a certain point. Similarly, rat brains first rendered thermotolerant appeared less vulnerable to ischemia-reperfusion-induced damage as well as to the deleterious effects of glutamate stimulation (23). In rodents first subjected to hyperthermia, the extent of retinal damage due to a subsequent intense light exposure was greatly diminished. Moreover, these investigators showed that maximal protection conferred correlated nicely with the overall levels of the highly stress-inducible hsp 72 which accumulated in the retina after the priming heat shock treatment (28). Lastly, in a rat model of the human adult respiratory distress syndrome, rats first made thermotolerant (via whole body hyperthermia) suffered no mortality as compared to $27 \%$ mortality observed for the nonheated control group (29).

Results such as these have stimulated efforts at possibly harnessing the protective effects of the thermotolerant phenotype in clinically relevant situations. One important issue is 
how to develop the thermotolerant phenotype in a timely and clinically relevant fashion. In vitro, full development of the tolerant phenotype requires anywhere from 8-18 $\mathrm{h}$ after the initiation of the priming stress treatment, likely the time period necessary to synthesize and accumulate maximal levels of the stress proteins. In the case of the heart, it is not possible to predict the occurrence of an acute myocardial infarction. Those individuals at high risk for an imminent infarction are typically already experiencing severe ischemia. The extent to which these ischemic episodes in humans induce a stress response remains an open question. Even if a pharmacological means of rapidly inducing the thermotolerant phenotype (on the order of minutes to perhaps a few hours) is developed, the applicability to acute clinical situations is unclear. Application of the stress response to clinical medicine is more promising in nonemergent situations such as scheduled surgery. For example, reconstructive surgeons have found that skin flap survival improves if the flap is first made thermotolerant (30). Other possible applications include making donor transplant organs thermotolerant and thereby possibly increasing the window of time that the organ can be transplanted and/or possibly even improving survivability once transplanted. The enthusiasm for exploiting the stress response in clinical medicine must be tempered, however, by the paucity of information regarding the price the cell pays for becoming thermotolerant. In particular, we still do not know all of the other cellular consequences (e.g., effects on cell cycle, ability of the cell to respond to other stimuli etc.) associated with the acquisition of the thermotolerant phenotype.

The recent evidence that the expression of the stress proteins also may be regulated, at least in part, by neuroendocrine mechanisms represents an exciting new development. Activation of the hypothalamic-pituitary axis (using a rodent "restraint" model) was shown to induce specific expression of the most highly stress induced protein, hsp72, in the rat adrenal cortex. Hypophysectomy ablated the response, and the addition of adrenocorticotropic hormone restored specific expression in the hypophysectomized rats. In contrast, in the same model system the sympathetic nervous system appeared to be important in the regulation of both hsp72 and hsp28 expression in the rat aorta. Adrenergic antagonists were found to block such expression, whereas adrenergic agonists induced their expression $(34,35$, 36). Lastly, a dopamine agonist induced expression of hsp72 in both the adrenal cortex and aorta of the rat (37). Clearly, these findings suggest that adrenergic, and dopaminergic agents, as well as drugs that affect the hypothalamic-pituitary axis, have potential as modulators of the heat shock response in humans.

\section{Malignancy}

The ability to modulate the stress response also has therapeutic implications as it relates to cancer. The increased expression of hsp28 and hsp72 has been associated with enhanced survival of tumor cells subjected to some cancer chemotherapeutic agents (for review see reference 31 ). The increased expression of the multidrug resistance protein (MDR), whose corresponding gene contains an appropriate heat shock element, has been clearly shown to underlie the development of resistance to many cancer chemotherapeutic agents (for review see reference 32 ). Thus, finding a way by which to down regulate or even prevent the expression of stress proteins in malignant cells may enhance the efficacy of many chemotherapeutic agents. Such an advance also might allow the use of lower doses of chemotherapy, and thus perhaps minimize the toxic side effects of these agents on normal cells. In preliminary experiments, a flavonoid compound querecetin, reported to be an inhibitor of protein kinases, was shown to specifically inhibit the expression of the stress proteins, although the mechanism by which such inhibition is manifested is not known (33).

\section{Immune system}

Investigation of the possible clinical implications of the stress response/proteins gained added impetus as evidence began to accumulate that one or more of the stress proteins play a role in various aspects of the immune system (for review see references 6 and 38). First, genes encoding two members of the hsp70 family were found to reside within the MHC. In this regard, computer modeling of the three-dimensional structure of the carboxy-terminal domain of hsp70, that region in the protein thought to be involved in the binding of both small peptides as well as unfolded polypeptides, revealed a possible peptide binding motif very similar to the peptide binding cleft of the MHC class I proteins. Second, a peptide binding protein, termed PBP 74, was identified and shown to be related to the other members of the hsp 70 family. PBP 74 has been proposed be involved in peptide loading of MHC class II molecules. Third, deoxyspergulain, an immunosuppressant agent whose mechanism of action is unknown, but appears distinct from that of both cyclosporin A and FK506, was found to specifically bind to hsp73 (39). As mentioned earlier, FK506 appears to bind to hsp56 (a protein with rotamase activity) and together the complex appears to have immunosuppressive activity (8). Finally, 2 of the 11 self peptides isolated from purified class I HLA-B27 were shown to be peptides derived from hsp90. This latter result raises the question as to whether the immune system constantly is presented with peptides derived from "self " stress proteins as a part of the mechanism for monitoring the presence of stress proteins from invading microorganisms? (and discussed further below). While these types of observations clearly are intriguing, they remain primarily phenomenological in nature and therefore, will require further study to ascertain their biological relevance.

\section{Infectious diseases}

More compelling and scientifically developed is the observation that stress proteins from a variety of pathogens act as immunodominant antigens in animals (for reviews see references 40 , 41 , and see Table II). For many years, it had been known that bacteria produced an $\sim 60-\mathrm{kD}$ genus specific protein; an antibody raised against this protein from one species tended to recognize the protein in all other species of the genus, but not in any species from another genus. This protein was shown to be GroEL, the bacterial homologue of hsp60, and is a major target of the mammalian humoral response to bacterial infections. Interestingly in many parasitic infections it is the parasitic form of hsp70 and in some cases hsp90 which represents a major target for the humoral arm of the immune response. Recent evidence indicates that at least some parasitic and bacterial stress proteins can also induce a relatively strong $\mathrm{T}$ cell response. Moreover, roughly 10-20\% of gamma/delta T cells, a poorly understood population of $\mathrm{T}$ cells, have been shown to be specific for stress proteins of various pathogens (42). A particularly attractive hypothesis is that this class of $\mathrm{T}$ cells, 
Table II. Heat Shock Proteins Are Immunodominant Antigens of Infectious Organisms*

\begin{tabular}{ll}
\hline \multicolumn{1}{c}{ Disease } & \multicolumn{1}{c}{ Infectious agent } \\
\hline Tuberculosis & Mycobacterium tuberculosis \\
Leprosy & Mycobacterium leprae \\
Schistosomiasis & Schistosoma mansoni \\
Malaria & Plasmodium falciparum \\
Chagas & Trypanosoma cruzi \\
Legionnaires & Legionella pneumophila \\
Syphillis & Treponema pallidum \\
Blinding Trachoma & Chlamydia trachomatis \\
Lyme & Borrelia burgdorferi \\
Q fever & Coxiella burnetti \\
Ocular filariasis & Onchocerca volvulus \\
Lymphatic filariasis & Brugia malay \\
\hline
\end{tabular}

* Summarized from the literature.

which by lining the airway, gut, and epidermal epithelium, are well positioned to provide an early line of immune defense at major body-environment interfaces where pathogen entry into the host is likely to occur.

Does the immune response to bacterial and parasitic stress proteins protect the host from infection? A number of studies suggest that the answer is yes. Protection against chlamydial diseases was associated with an immune response to chlamydial DnaK, the bacterial homologue of hsp70 (43). In a guinea pig model of Legionnaire's disease, immunization with GroEL purified from Legionella pneumophila was effective in preventing disease (44). Immunization of mice with an $80-\mathrm{kD}$ protein from Histoplasma capsulatum, a protein related to hsp70, resulted in improved resistance to infection (45). In a monkey model of malaria, immunization with Plasmodium falciparum hsp70 prevented infection with blood stages of $P$. falciparum (46). In addition, infected hepatocytes appeared to express a cell surface epitope from this same hsp70. An antihsp70 antibody recognizing this epitope appeared effective in an antibody-dependent cytolysis of the infected hepatocyte (47). Thus, these examples indicate that the immune response to stress proteins from various infectious pathogens is associated with protection for the host.

Why does the immune system appear to preferentially target the stress proteins of infectious pathogens? Several possible reasons that are not mutually exclusive have been suggested $(38,40,41)$. The stress proteins represent a relatively abundant set of proteins within the invading pathogen, and therefore, on a purely statistical basis, the immune system may be more likely to recognize these "foreign" proteins rather than a less abundant one. In this regard it also has been reported that infection not only induces a stress response in the host, but also in the invading pathogen (thereby increasing the levels of "pathogenic stress proteins") (48). Alternatively, faced with a vast number of potential pathogens, the immune system may have simplified the problem of detection by taking advantage of the fact that stress proteins are essential components of any organism (i.e., most of the genes encoding the stress proteins are essential for growth) and they exhibit a high degree of homology. For example, recognizing genus specific GroEL may have enabled the immune system to strike a balance between the need for sensitivity (i.e., the ability to quickly recognize the presence of a pathogen), and the need for specificity (i.e., responding only to pathogenic bacteria as opposed to normal commensal flora such as exists within the intestines). Similarly, by preferentially recognizing parasitic hsp70, the immune system can distinguish between a bacterial versus a fungal infection.

The association of a protective humoral response against pathogenic stress proteins lies at the crux of an apparent conundrum. Specifically, the vast majority of studies have shown that the different stress proteins are located inside the cell. Although still requiring further study, there have been a number of reports which indicate that in some instances mycobacterial/bacterial stress proteins either may be secreted or present on the bacterial membrane. If however they are entirely intracellular, how does the humoral response confer protection? One mechanism could be the earlier described antibody-dependent cell cytolysis of hepatocytes infected with $P$. falciparum which present the pathogen's hsp70 on the surface of the infected cell. Yet, many bacterial and parasitic pathogens produce their deleterious effects without ever entering host cells. One intriguing study suggested that GroEL from Salmonella typhimurium mediated binding of the bacterium to intestinal mucus (49). While GroEL appeared to be secreted, it apparently was also present on the cell surface. Interestingly, antibodies against GroEL blocked Salmonella aggregation on the intestinal mucus. No biochemical evidence was presented demonstrating a direct interaction between GroEL and the previously identified $15-\mathrm{kD}$ glycoprotein component of intestinal mucus that mediated binding of S. typhimurium. Nevertheless, this report suggests that GroEL, by a change in its locale to the cell surface and/or its secretion, may act as a "virulence factor" in S. typhimurium infection. Other pathogens may have evolved similar mechanisms of infection which may account for the observation that the humoral immune response to stress proteins has been associated with protection. Indeed, chlamydial DnaK has been observed on the surface of elementary bodies, although its function at this site is unclear (43). As mentioned earlier, antibodies against chlamydial DnaK were associated with protection from the disease.

What about cell-mediated immunity against pathogenic stress proteins? Most bacterial and parasitic agents appear to induce such an immune response, but in some cases, rather than protecting the host, the response tends to exacerbate or contribute to the disease. For example, $T$ cell responses to chlamydial GroEL has been associated with infection of the female reproductive tract as well as the respiratory system (50). Similarly, T cells that recognize GroEL from Borrelia burgdorferi, the etiologic agent of Lyme's disease, line the synovium of joints affected by the arthritic component of the disease (51). These types of observations have raised the question of whether these $\mathrm{T}$ cells were part of a general inflammatory response in these illnesses, or instead, directly caused damage, perhaps by cross-reacting with self-stress proteins. If the latter were true, it would imply that the well conserved stress proteins contribute in some way to autoimmune diseases.

\section{Autoimmune diseases}

The idea that stress proteins play some role in different autoimmune diseases remains highly controversial (52). There are a few animal studies whose results are suggestive but by no means 
support, a direct role for stress proteins in autoimmune disease. For example, $\mathrm{T}$ cell reactivity to mycobacterial GroEL appeared to be important in the development of disease in the non-obese diabetic (NOD) mouse model of insulin-dependent diabetes (for review see reference 53). More recent work, however, suggested that these GroEL reactive $T$ cells somehow modulated the immune response rather than directly causing diabetes in the NOD mouse $(54,55)$. Those $T$ cells that recognized mycobacterial GroEL in the adjuvant-induced arthritis rat model probably play a similar modulatory role (for review see reference 53). With regards to human autoimmune diseases and stress proteins, other than juvenile chronic arthritis (JCA), most studies have demonstrated guilt only by association. To date, only the synovial $\mathrm{T}$ cells from patients with JCA have been shown to respond strongly to human hsp60 (56). Otherwise, the literature is filled with studies that are mainly of a phenomenological nature; either they refute (several) the presence of an association between stress proteins and most human autoimmune diseases; or they confirm an association (many), but do not answer the fundamental question of cause and effect.

The discovery that $T$ cells reactive to self-stress proteins are present normally in otherwise healthy individual led to the proposal that these $\mathrm{T}$ cells may recognize other cells undergoing a stress response (for example, due to some type of an infection or transformation) and thereby help to eliminate these cells (38). Whether a particular stress event, be it infection, transformation, or some other type of metabolic insult, results in the processing and presentation of peptides derived from self-stress proteins to the immune system remains an extremely interesting but unanswered question. Studies with established cell lines have demonstrated that the intracellular locale of many stress proteins changes in response to stress, but localization to the cell surface, either as an intact protein or as a peptide associated with the histocompatibility complex, has not been clearly demonstrated. However those observations mentioned earlier in which two of eleven self peptides present within the MHC class I molecule were derived from hsp90 implies that cells may normally present self peptides derived from stress proteins on a routine basis. That different forms of stress in vivo may lead to an increase and/or alteration in the presentation of self-stress peptides thereby providing for some type of activating signal to the immune system is an interesting question which deserves additional study.

Further fueling the idea that stress proteins are important in immune cell recognition are studies reporting that both grp94 and hsp73 behaved as tumor rejection antigens. Both of these stress proteins, when purified from a particular tumor and subsequently used as an immunogen conferred protection to challenge from that same tumor but not to an antigenically distinct tumor. Grp94 and hsp73 from normal cells provided no such tumor immunity, and sequence analysis of the purified stress proteins isolated from the tumors revealed no differences when compared to the proteins isolated from nontumorigenic cells. Subsequent work however, reported that both hsp73 and grp94 bound to a heterogeneous population of peptides $(57,58)$. Presumably, the associated peptides found with grp94 and hsp 73 from the tumor cells were responsible for the successful tumor immunity, but the direct demonstration that the bound peptides, when used as the immunogen, could confer tumor resistance remains to be shown. Thus, these data suggest that stress proteins are involved in processing proteins for antigenic presentation, rather than being immunogenic themselves.

\section{Vaccines and immunotherapy}

There is increasing excitement and enthusiasm for the use of mycobacterial and parasitic forms of the stress proteins as novel acellular vaccines and/or carrier-free adjuvants. For example, mice primed with BCG and then immunized with a hapten conjugated to tuberculin PPD produced long lasting and high titres of anti-hapten antibodies without the use of adjuvants (59). The same effect was observed if the hapten was conjugated directly to Mycobacterial GroEL or DnaK. Importantly, this effect occurred even in the animal showing high titres of antibody against the mycobacterial GroEL or DnaK stress proteins used as the adjuvant. In fact, high doses of GroEL was shown to be as effective a primer as BCG. However, the most provocative and exciting finding was that an effective $\mathrm{T}$ cellmediated response could be induced by hapten conjugated to DnaK, without the need for either an adjuvant or for previous priming (60). Clearly, these results have broad implications for vaccine development against infectious diseases and tumors.

\section{Toxicology}

Although too extensive in scope to adequately present here, we should mention briefly that changes in stress protein expression may prove useful as it relates to toxicology. In the hopes of developing rapid assays as well as reducing the use of animals, toxicologists are exploring the use of changes in the expression of one or more of the stress proteins, in cells grown in vitro, as a sensitive and reliable indicator of the possible toxic effects of different compounds. As a further extension of this type of technology, investigators are developing transgenic stress reporter organisms. Using well defined heat shock promoter elements to drive the expression of a reporter gene (luciferase, B-galactosidase, chloramphenicol acetyl transferase) the reporter organism carrying such a construct would be used, for example, in the monitoring of environmental pollutants (61). Although such approaches are still at an exploratory stage and requiring substantial validation efforts, exploiting changes in the expression of the stress proteins as well as other gene products associated with cellular injury (metallothionines, cytochrome 450 system) may revolutionize the field of toxicology.

\section{Summary}

A field of research that began with a curious observation in Drosophila has resulted in a new understanding of how cells respond to sudden and adverse changes in their environment. In addition through the study of the structure/function of the stress proteins, especially those which function as molecular chaperones, new insights into the details by which proteins are synthesized and acquire their final biologically active conformation have been realized. Equally exciting is the progress being made as it relates the potential diagnostic and therapeutic applications of the stress-response proteins. The use of stress proteins as the next generation of vaccines and/or their use as potentially powerful adjuvants, capable of stimulating both $T$ and $B$ cell responses to a particular antigen of interest appear close to becoming a reality. One wonders how many more surprises are in store for us as we continue to explore this evolutionally conserved cellular stress response. 


\section{Acknowledgments}

We thank members of the lab for reviewing the manuscript at various stages of preparation; Joel Ernst and David Erle for critical reading of the final draft; and Christine Mok for secretarial assistance.

G. Minowada has received past fellowship support from the Cardiovascular Research Institute (NIH HL-07185) and presently is supported by GM 15526 . W. J. Welch is supported by National Institutes of Health grant GM-33551, National Science Foundation grant MCB9018320, and American Cancer Society grant CB-91A.

\section{References}

1. Hightower, L. E. 1980. Cultured animal cells exposed to amino acid analogues or puromycin rapidly synthesize several polypeptides. J. Cell. Physiol 102:407-424.

2. Anathan, J., A. L. Goldberg, and R. Voellmy. 1986. Abnormal proteins serve as eukaryotic stress signals and trigger the activation of heat shock genes. Science (Wash. DC). 232:252-254.

3. Lis, J., and C. Wu. 1993. Protein traffic on the heat shock promoter: parking, stalling, and trucking along. Cell. 74:1-4.

4. Sistonen, L., K. D. Sarge, B. Phillips, K. Abravaya, and R. I. Morimoto. 1992. Activation of heat shock factor 2 during hemin-induced differentiation of human erythroleukemia cells. Mol. Cell. Biol. 12:4104-4111.

5. Lindquist, S. C. 1986. The heat shock response. Annu. Rev. Biochem. 55:1151-1191.

6. Welch, W. J. 1992. Mammalian stress response: cell physiology, structure/ function of stress proteins, and implications for medicine and disease. Physiol. Rev. 72:1063-1081.

7. Pratt, W. B. 1993. The role of heat shock proteins in regulating the function, folding, and trafficking of the glucocorticoid recepto. J. Biol. Chem. 268:2145521458

8. Yem, A. W., A. G. Tomasselli, R. L. Heinrikson, H. Zurcher-Neely, V. A Ruff, R. A. Johnson, and M. R. Deibel, Jr. 1992. The hsp56 component of steroid receptor complexes binds to immobilized FK506 and shows homology to FKBP12 and FKBP-13. J. Biol. Chem. 267:2868-2871.

9. Sanchez, E. R. 1990. Hsp56: A novel heat shock protein associated with untransformed steroid receptor complexes. J. Biol. Chem. 265:22067-22070.

10. Hartl, F. U., J. Martin, and W. Neupert. 1992. Protein folding in the cell: the role of chaperones hsp70 and hsp 60. Annu. Rev. Biophys. Biomol. Struct. 21:293-322.

11. Georgopoulos, C., and W. J. Welch. 1993. Role of the major heat shock proteins as molecular chaperones. Annu. Rev. Cell Biol. 9:601-634.

12. Langer, T., G. Pfeifer, J. Martin, W. Baumeistere, and F. U. Hartl. 1992. Chaperonin-mediated protein folding: GroES binds to one end of the GroEL cylinder, which accomodates the protein within its central cavity. EMBO (Eur Mol. Biol. Organ.) J. 11:4757-4765.

13. Kubota, H., G. Hynes, A. Canne, A. Ashworth, and K. Willison. 1994 Identification of six TCP-1 related genes encoding divergent subunits of the TCP1 containing chaperonin. Curr. Biol. 4:89-99.

14. Anfinsen, C. B. 1973. Principles that govern the folding of protein chains Science (Wash. DC). 8:223-230.

15. Ellis, R. J., and S. M. van der Vies. 1991. Molecular chaperones. Annu. Rev. Biochem. 60:321-347.

16. Brugge, J. S. 1986. Interaction of the Rous sarcoma Virus protein, pp60 $\mathrm{scc}$ with the cellular proteins pp50 and pp90. Curr. Top. Microbiol. Immunol. 123:1 23.

17. Smith, D. F., and D. O. Toft. 1993. Steroid receptors and their associated proteins. Mol. Endocrinol. 7:4-11.

18. Jakob, U., M. Gaestel, K. Engel, and J. Buchner. 1993. Small heat shock proteins are molecular chaperones. J. Biol. Chem. 268:1517-1520.

19. Miron, T., K. Vancompernnolle, J. Vandkerckhove, M. Wilchek, and B. A Geiger. 1991. 25-kD inhibitor of actin polymerization is a low molecular mass heat shock protein. J. Cell Biol. 114:255-261.

20. Lavoie, J. N., G. Gingras-Breton, R. M. Tanguay, and J. Landry. 1993. Induction of chinese hamster hsp27 gene expression in mouse cells confers resistance to heat shock; hsp 27 stabilization of the microfilament organization. $J$. Biol. Chem. 268:3420-3429.

21. Lavoie, J. N., E. Hickey, L. A. Weber, and J. Landry. 1993. Modulation of actin microfilament dynamics and fluid phase pinocytosis by phosphorylation of heat shock protein 27. J. Biol. Chem. 268:24210-24214.

22. Vass, K., M. L. Berger, T. S. J. Nowak, W. J. Welch, and H. Lassmann. 1989. Induction of stress protein Hsp 70 in nerve cells after status epilepticus in the rat. Neurosci. Lett. 100:259-264.

23. Sloviter, R. S., and D. H. Lowenstein. 1992. Heat shock protein expression in vulnerable cells of the rat hippocampus as an indicator of excitation-induced neuronal stress. J. Neurosci. 12:3004-3009.

24. Delcayre, C. J., L. Samuel, F. Marotte, F. Best-Belpomme, J. J. Mercadier, and L. Rappaport. 1988. Synthesis of stress proteins in rat cardiac myocytes 24 days after the imposition of hemodynamic overload. J. Clin. Invest. 82:460468 .

25. Donnelly, T. J., R. E. Sievers, F. L. J. Vissern, W. J. Welch, and C. L. Wolfe. 1992. Heat shock protein induction in rat hearts: a role for improved myocardial salvage after ischemia and reperfusion? Circulation. 85:769-778.

26. Currie, R. W., R. M. Tanguay, and J. G. Kingma. 1993. Heat shock response and limitation of tissue necrosis during occlusion/reperfusion in rabbit hearts. Circulation. 87:963-971.

27. Yellon, D., M. E. Miodromitis, D. S. Latchman, D. M. Van Winkle, J. M. Downey, F. M. Williams, and T. J. Williams. 1992. Whole body heat stress fails to limit infarct size in the reperfused rabbit heart. Cardiovasc. Res. 26:342-346.

28. Barbe, M. F., M. Tytell, D. J. Gower, and W. J. Welch. 1988. Hyperthermia protects against light damage in the rat retina. Science (Wash. DC). 241:18171820.

29. Villar, J., J. D. Edelson, M. Post, B. Mullen, and A. Slutsky. 1993 Induction of heat stress proteins is associated with decreased mortality in an animal model of acute lung injury. Am. Rev. Respir. Dis. 147:177-181.

30. Koenig, W. J., R. A. Lohner, G. A. Perdrizet, M. E. Lohner, R. T Schweitzer, and V. L. Lewis, Jr. 1992. Improving acute skin-flap survival through stress conditioning using heat shock and recovery. Plast. Reconstr. Surg. 90:659664

31. Hahn, G. M., and G. C. Li. 1990. Thermotolerance, thermoresistance, and thermosensitization. In Stress Proteins in Biology and Medicine. R. I. Morimoto, A. Tissieres, and C. Georgopoulos, editors. Cold Spring Harbor Laboratory Press, Cold Spring Harbor, New York. 79-100.

32. Gottesman, M. M., and I. Pastan. 1988. The multidrug transporter, a double-edged sword. J. Biol. Chem. 263:12163-12166.

33. Hosokawa, N., K. Hirayoshi, A. Nakai, Y. Hosokawa, N. Marui, M. Yoshida, T. Sakai, H. Nishino, A. Aoike, K. Kawai, and K. Nagata. 1990. Flavonoids inhibit the expression of heat shock proteins. Cell Struct. Funct. 15:393401.

34. Blake, M. J., R. Udelsman, G. J. Feulner, D. D. Norton, and N. J. Holbrook. 1991. Stress induced heat shock protein 70 expression in adrenal cortex: an adrenocorticotropic hormone-sensitive, age-dependent response. Proc. Natl. Acad. Sci. USA. 88:9873-9877.

35. Udelsman, R., M. J. Blake, and N. J. Holbrook. 1991. Molecular response to surgical stress: specific and simultaneous heat shock protein induction in the adrenal cortex, aorta, and vena cava. Surgery. 110:1125-1131.

36. Udelsman, R., M. J. Blake, C. A. Stagg, D-G. Li, D. J. Putney, and N. J. Holbrook. 1993. Vascular heat shock protein expression in response to stress. Endocrine and autonomic regulation of this age-dependent response. J. Clin. Invest. 91:465-473.

37. Blake, M. J., D. J. Buckley, and A. R. Buckley. 1993. Dopaminergic regulation of heat shock protein-70 expression in adrenal gland and aorta. Endocrinology. 132:1063-1070.

38. DeNagel, D. C., and S. K. Pierce. 1993. Heat shock proteins in immune responses. Crit. Rev. Immunol. 13:71-81.

39. Nadler, S. G., M. A. Tepper, B. Schacter, and C. E. Mazzucco. 1992. Interaction of the immunosuppressant Deoxyspergualin with a member of the hsp70 family of heat shock proteins. Science (Wash. DC). 258:484-486.

40. Young, R. A., and T. J. Elliot. 1989. Stress proteins, Infection, and Immune Surveillance. Cell. 59:5-8.

41. Young, D. B. 1992. Heat-shock proteins: immunity and autoimmunity. Curr. Opin. Immunol. 4:396-400.

42. Born, W., M. P. Happ, A. Dallas, C. Reardon, R. Kubo, T. Shinnick, P. Brennan, and R. O'Brien. 1990. Recognition of heat shock proteins and gamma/ delta function. Immunol. Today. 11:1-4.

43. Zhong, G., and R. C. Brunham. 1992. Antigenic analysis of the chlamydial 75-kilodalton protein. Infect. Immun. 60:323-328.

44. Blander, S. J., and M. A. Horwitz. 1993. Major cytoplasmic membrane protein of Legionella pneumophila, a genus common antigen and member of the hsp60 family of heat shock proteins, induces protective immunity in a guinea pig model of Legionnaire's disease. J. Clin. Invest. 91:717-723.

45. Gomez, F. J., A. M. Gomez, and G. S. Deepe, Jr. 1992. An 80-kilodalton antigen from Histoplasma capsulatum that has homology to heat shock protein 70 induces cell-mediated immune responses and protection in mice. Infect. Immun. 60:2565-2571.

46. Dubois, P., J. P. Dedet, T. Fandeur, C. Roussilhon, M. Jendoubi, S. Pauillac, O. Mercereau-Puijalon, and L. Pereira da Silva. 1984. Protective immunization of the squirrel monkey against asexual blood-stages of Plasmodium falciparum by use of parasite protein fractions. Proc. Natl. Acad. Sci. USA. 81:229232.

47. Renia, L., D. Mattei, J. Goma, S. Pied, P. Dubois, F. Miltgen, A. Nussler, H. Matile, F. Menegaux, M. Gentilini, and D. Mazier. 1990. A malaria heat- 
shock-like determinant expressed on the infected hepatocyte surface is the target of antibody dependent cell-mediated cytotoxic mechanisms by non-parenchymal liver cells. Eur. J. Immunol. 20:1445-1451.

48. Buchmeier, N. A., and F. Heffron. 1990. Induction of Salmonella stress proteins upon infection of macrophages. Science (Wash. DC). 248:730-732.

49. Ensgraber, M., and M. Loos. 1992. A 66-kilodalton heat shock protein of Salmonella typhimurium is responsible for binding of the bacterium to intestinal mucus. Infect. Immun. 60:3072-3078.

50. Morrison, R. P., R. J. Belland, K. Lyng, and H. D. Caldwell. 1989. Chlamydial disease pathogenesis: the $57-\mathrm{kD}$ chlamydial hypersensitivity antigen is a stress response protein. J. Exp. Med. 170:1271-1283.

51. Shanafelt, M-C., P. Hindersson, C. Soderberg, N. Mensi, C. W. Turck

D. Webb, H. Yssel, and G. Peltz. 1991. T cell and antibody reactivity with the Borrelia burgdorferi $60 \mathrm{kDa}$ heat shock protein in Lyme arthritis. J. Immunol. 146:3985-3992.

52. Kaufmann, S. H. E. 1991. Heat shock proteins and autoimmunity: fact or fiction? Curr. Biol. 1:359-361.

53. Cohen, I. R. 1991. Autoimmunity to chaperonins in the pathogenesis of arthritis and diabetes. Annu. Rev. Immunol. 567-589.

54. Kaufman, D. L., M. Clare-Salzer, J. Tian, T. Forsthuber, G. S. P. Ting, P. Robinson, M. A. Atkinson, E. E. Sercarz, A. J. Tobin, and P. V. Lehmann 1993. Spontaneous loss of T-cell tolerance to glutamic acid decarboxylase in murine insulin-dependent diabetes. Nature (Lond.) 365:69-72.
55. Tisch, R., X-D. Yang, S. M. Singer, R. S. Liblau, L. Fugger, and H. O McDevitt. 1993. Immune response to glutamic acid decarboxylase correlates with insulitis in non-obese diabetic mice. Nature (Lond.) 365:72-75.

56. De Graeff-Meeder, E. R., R. Van Der Zee, G. T. Rijkes, H-J. Schuurman, W. Kuis, J. M. J. Bijlsma, B. J. M. Zegers, and W. Van Eden. 1991. Recognition of human $60 \mathrm{kD}$ heat shock protein by mononuclear cells from patients with juvenile chronic arthritis. Lancet. 337:1368-1372.

57. Li, Z., and P. K. Srivastava. 1993. Tumor rejection antigen gp96/grp94 is an ATPase: implications for protein folding and antigen presentation. EMBO (Eur. Mol. Biol. Organ.) J. 12:3143-3151.

58. Udono, H., and P. K. Srivastava. 1993. Heat shock protein 70 -associated peptides elicit specific cancer immunity. J. Exp. Med. 178:1391-1396.

59. Lussow, A. R., C. Barrios, J. van Embden, R. Van der Zee, A. S. Verdini, A. Pessi, J. A. Louis, P-H. Lambert, and G. Del Guidice. 1991. Mycobacterial heat-shock proteins as carrier molecules. Eur. J. Immunol. 21:2297-2302.

60. Barrios, C. A., R. Lussow, J. Van Embden, R. Van der Zee, R. Rappuoli, P. Constantino, J. A. Louis, P-H. Lambert, and G. Del Guidice. 1992. Mycobacterial heat shock proteins as carrier molecules. II. The use of the $70 \mathrm{kDa}$ mycobacterial heat shock proteins as carrier for conjugated vaccines can circumvent the need for adjuvants and Bacillus Calmette Guerin priming. Eur. J. Immunol. 22:1365-1372.

61. Welch, W. J. 1993. How cells respond to stress. Scientific American. 268:56-62. 